\title{
El archivo municipal de Monforte del Cid y la e- administración: una visión de futuro
}

\section{The municipal archive of Monforte del Cid and the e- administration: a vision for the future}

\author{
Inmaculada Mayor González \\ inmamay@gmail.com
}

Técnico de archivo del ayuntamiento de Monforte del Cid

\section{Resumen}

El presente artículo recoge la experiencia del servicio de archivo de una población de menos de 10.000 en la implantación de la Administración electrónica. Se centra en el archivo municipal como servicio responsable de la puesta en marcha de la Administración electrónica en el ayuntamiento de Monforte del Cid (Alicante), identificando ventajas y carencias del sistema implantado y cómo afecta a los archivos

\section{Palabras clave}

Acceso al documento electrónico; Administración electrónica; Archiveros; Archivos electrónicos; Archivos municipales; Documento electrónico; Legislación archivística; Preservación digital; Recursos electrónicos; Soportes de información electrónica

\begin{abstract}
This article presents the experience of archival service a town of less than 10,000 population in the implementation of e-Administration. It focuses on the municipal archive as the service responsible for its implementation in the city hall of Monforte del Cid (Alicante), identifying advantages and shortcomings of the system implemented and how it affects the archives
\end{abstract}

\section{Keywords}

Acces to the electronic record; Archival legislation; Archivists; Digital preservation; e-Administration; Electronic information supports; Electronics archives; Electronic records; Electronic resources; Municipal archives 
Recibido: $15 / 11 / 2017$

Aceptado: 19/12/2017

DOI: http://dx.doi.org/10.5557/IIMEI8-N15-125138

Descripción propuesta: Mayor González, Inmaculada, 2017. El archivo municipal de Monforte del Cid y la e-administración: una visión de futuro. Métodos de información, 8(15), pp. 125-138.

\section{Introducción}

\subsection{Monforte del Cid}

El municipio de Monforte del Cid está ubicado en la comarca del Medio Vinalopó, con una población entorno a los 8.000 habitantes. Se sitúa a $20 \mathrm{~km}$. de Alicante, en la autovía que une la costa levantina con Madrid y a corta de distancia de otras grandes poblaciones alicantinas como Elche o Elda, por lo que hacen de nuestro municipio un lugar privilegiado desde un punto de vista geográfico. Está situado en una comarca agrícola y marmolera, siendo el máximo exponente de su actividad agraria la uva de mesa con denominación de origen "Uva de Mesa Embolsada del Vinalopó".

Respecto a la historia de Monforte del Cid, de alguna manera viene marcada por su situación geográfica, ya que nuestra población ha visto pasar culturas y civilizaciones diversas a lo largo de los siglos. Vestigios de ese pasado son los restos arqueológicos íberos y romanos conservados en Museo de Historia de la Villa IBERO, donde destacan los toros ibéricos. También debemos resaltar el casco antiguo de origen medieval con la morería y la Iglesia en alto, ubicada en el lugar donde se levantaba el antiguo castillo de Monfort ${ }^{1}$, y la pedanía de Orito, centro importante de peregrinaje de la provincia de Alicante desde hace siglos.

Por último señalar también nuestra población como cuna de personajes históricos, como Jorge Juan Santacilia, ilustre marino y científico natural de Monforte. 


\subsection{Historia del archivo}

Respecto al Archivo de Monforte del Cid, debemos decir que las primeras referencias a la existencia del mismo las encontramos en las actas de los Cabildos de finales del siglo XVIII y principios del XIX. No sabemos su ubicación en aquella época, pero probablemente siempre haya estado en la Casa Consistorial, donde se encuentra hoy el Archivo Administrativo. En cualquier caso, podríamos remontar el origen del Archivo a la creación del Consell de Monfort, hacia el s. XV, época de la que datan los primeros documentos custodiados en el Archivo Histórico. El primer inventario realizado en papel y del que disponemos del original es del año 1930; el último elaborado en papel y en fichas, y que posteriormente fue informatizado, es de 1991. Pero será en el 2001 cuando se cree el servicio de Archivo, con un responsable cualificado a tiempo completo encargado tanto del archivo administrativo como del histórico.

En definitiva, podríamos decir, que la historia del Archivo de Monforte del Cid es la de sus instituciones, ya que refleja la evolución de éstas, en la medida en que custodia toda la documentación producida por la Corporación Municipal a lo largo del tiempo.

\subsection{Objetivos y metodología}

En el siguiente trabajo hemos tratado de desglosar la relación/ubicación del servicio de archivo en la implantación de la e-Administración en el ayuntamiento de Monforte del Cid, por medio del desarrollo de los siguientes puntos:

-Ubicación del servicio de archivo, su historia y organización, como punto de partida para poder clarificar su situación en la actualidad.

-Experiencia del archivo municipal en la implantación de la Administración Electrónica en el ayuntamiento de Monforte del Cid desde su puesta en marcha, desarrollando su materialización a través de la legislación vigente, la adhesión al Plan Moderniza 6.0 y el uso de la plataforma Gestiona.

\section{E1 Archivo Municipal de Monforte del Cid}

El Archivo Municipal de Monforte del Cid organiza y custodia la documentación municipal. Sus funciones abarcan desde la gestión administrativa hasta la organización, conservación y difusión de la 
documentación histórica. Dado que el archivo municipal de Monforte del Cid pertenece a un municipio de menos de 10.000 habitantes, no gestiona solo el archivo histórico, sino que también organiza y conserva el archivo administrativo, abarcando incluso el archivo de gestión (dada la escasez de espacio y medios humanos).

En este sentido, en el proceso de cambio hacia la e-Administración y dada la presencia del archivo prácticamente en todo el ciclo vital del documento, este servicio no podía quedarse al margen de la implantación del nuevo entorno digital en el ayuntamiento. De hecho, ha sido el encargado de poner en marcha el proceso, a través de la Diputación Provincial de Alicante, como garante de la perdurabilidad y adecuada conservación de la documentación electrónica a lo largo del tiempo.

\subsection{Organización y descripción}

El archivo municipal de Monforte del Cid conserva la documentación de cualquier época y soporte, debidamente organizada para su uso en la gestión administrativa municipal, la información a los ciudadanos, la cultura y la investigación.

Queda configurado en función del ciclo vital de los documentos y del espacio disponible, por lo que la falta de éste va marcando las transferencias de un archivo a otro. Así distinguimos:

1. Archivo administrativo:

-El archivo de gestión: reúne documentos en trámite y expedientes ya tramitados sometidos a una continua utilización por parte de las oficinas que los produce. Con carácter general, el periodo de permanencia de la documentación en este archivo es de cinco años, pero debido a la falta de espacio no siempre puede respetarse este periodo cronológico, aunque resulta sencillo de controlar al estar informatizado. También cabe destacar, como ya hemos mencionado, el hecho de que el archivo de gestión no se encuentre bajo la responsabilidad directa de las distintas unidades administrativas. Este archivo se sitúa en la casa consistorial, cerca de las oficinas, por lo que para ahorrar espacio en los distintos despachos, se centraliza la documentación en el archivo

-El archivo central/intermedio: a los cinco años de la finalización de su trámite la documentación se transfiere a este depósito. Aquí se incluye el central e intermedio porque en la práctica las infraestructuras archivísticas no permiten 
la disposición de tantos locales y personal al servicio de cada uno de los archivos, ya que tan solo hay un responsable al frente del archivo municipal, y la gestión conjunta del archivo central e intermedio es factible, ya que usan los mismos instrumentos de control e información; la diferencia estribaría en la frecuencia de consulta

2. Archivo histórico:

-El archivo histórico: se transfiere desde el archivo intermedio la documentación que deba conservarse permanentemente al haber adquirido valor histórico.

\section{E1 Archivo Municipal y la e-Administración}

La Administración Electrónica ya es un hecho en la mayoría de nuestros ayuntamientos, una evolución en la gestión administrativa pública que está cambiando procesos, sistemas y mentalidades. Y el Archivo, como servicio final de ese proceso, adquiere una nueva dimensión en su papel de conversión a lo que denominaremos Archivo Electrónico.

Son muchas las cuestiones que se plantean a la hora de valorar el papel de los archivos en esta nueva concepción, incluso ha planeado sobre nuestras cabezas la idea de un futuro incierto de nuestra profesión, dada la disponibilidad inmediata de los expedientes tramitados de manera telemática. Pero si analizamos bien las características de los documentos electrónicos, son obvios los riesgos de la ausencia de una organización y descripción archivística, sobre todo en dos aspectos: la trazabilidad que genera un expediente y garantizar la conservación permanente de los documentos electrónicos, dada la dependencia absoluta de éstos de un entorno tecnológico en constante evolución.

No vamos a entrar a analizar en profundidad la legislación en relación a la Administración Electrónica, pero sí quisiera hacer un breve apunte en relación a la legislación estatal, sobre todo en relación a dos normativas: la Ley 39/2015, de 1 de octubre, del Procedimiento Administrativo Común de las Administraciones Públicas (LPACAP) y el Real Decreto 4/2010, de 8 de enero, por el que se regula el Esquema Nacional de Interoperabilidad (ENI).

La Ley 39/2015, de 1 de octubre, del Procedimiento Administrativo Común de las Administraciones Públicas regula de forma clara aspectos básicos para la plena implantación de la Administración electrónica, como son los sistemas de 
autentificación, con la separación entre identificación y firma electrónica; sistemas de sello electrónico reconocido, las transformaciones válidas papelelectrónico y viceversa, el expediente electrónico, etc. Cabe destacar también el artículo 17 donde se estable de la obligatoriedad de la Administración Pública de mantener un archivo electrónico único que garantice la autenticidad, integridad y conservación de los documentos correspondientes a procedimientos finalizados. Del mismo modo, la Ley 40/2015, de 1 de octubre, de Régimen Jurídico del Sector Público (LRJSP) regula en su artículo 46, el archivo electrónico de documentos, poniendo el énfasis en la fase activa del archivo.

Por otro lado, el Esquema Nacional de Interoperabilidad regulado en el Real Decreto 4/2010, de 8 de enero y establecido en el artículo 156, apartado 1, de la Ley 40/2015, de 1 de octubre, de Régimen Jurídico del Sector Público, que sustituye al apartado 1 del artículo 42 de la Ley 11/2007, de 22 de junio, de Acceso electrónico de los ciudadanos a los Servicios Públicos, dispone una serie de medidas organizativas y técnicas a adoptar por las administraciones públicas, encaminadas a garantizar la conservación de los documentos a lo largo de su ciclo de vida, medidas que incluyen la definición de políticas de gestión de documentos, el foliado electrónico de expedientes, la asignación de metadatos a los documentos, el uso de formatos estándares para la conservación y la migración a nuevos formatos de los documentos obsoletos.

La Norma Técnica de Interoperabilidad de Politica de gestión de documentos electrónicos hace hincapié en la valoración documental y en la conservación. No habla claramente de archivos electrónicos, pero sí de repositorios y la importancia de un adecuado expurgo.

Por último, la Norma Técnica de Interoperabilidad para el documento electrónico habla de tres componentes:

-Contenido, entendido como conjunto de datos o información del documento

-Firma electrónica

-Metadatos

Este último componente adquiere una nueva dimensión en relación a la recuperación de la información y por tanto en la organización archivística digital. 
Pero para tratar de manera tangible el tema que nos ocupa, vamos a exponer la experiencia en relación a la e-Administración del Ayuntamiento de Monforte del Cid.

\subsection{La Administración Electrónica en el Ayuntamiento de Monforte del Cid}

En primer lugar debemos destacar, por un lado, un doble intento de implantación desde dos perspectivas diferentes:

1. Creación de plataforma de gestión de expedientes personalizada (de nueva creación)

2. Adhesión al Plan de Modernización para ayuntamientos implantado por la Diputación Provincial de Alicante.

Y en segundo lugar, el hecho de que en ambos proyectos, iniciados por Secretaría, el Archivo Municipal haya sido el servicio en el que se ha delegado la implementación y puesta en marcha. Esto da una idea de la gestión archivística del municipio, con la presencia del archivo prácticamente desde el archivo de gestión, de ahí su visibilidad en todas las dependencias y departamentos del ayuntamiento.

El primer intento se realizó a través de una empresa de gestión documental que ya trabajaba con nosotros en el mantenimiento de otros programas. Está empresa junto con Secretaría y la técnico de archivo llegó a diseñar un programa de gestión de expedientes. Partiendo del registro de entrada, se pensó en la identificación de expedientes utilizando el cuadro de clasificación del archivo municipal, de tal forma que al iniciarse desde el registro, se identificara el expediente mediante el código de cuadro de clasificación correspondiente. Es evidente que para su implantación debíamos hacer un estudio previo de la idoneidad del inicio de expediente mediante su identificación usando el cuadro de clasificación. Para ello, desde el archivo, suministramos el cuadro junto a una serie de puntualizaciones a tener en cuenta. Una vez comprobada la idoneidad, el técnico de la empresa y la archivera diseñaron la plantilla de gestión de expedientes, siguiendo para ello las directrices de las Normas Técnicas de Interoperabilidad de expediente electrónico y de documentos.

La falta de medios técnicos con ordenadores obsoletos y la inexperiencia de sus creadores, hizo de este primer intento algo baldío, ya que aunque el 
sistema era completo y abarcaba todas las posibilidades, resultaba complejo y lleno de incertidumbres, ya que no partíamos de una plataforma de gestión de expedientes ya creada e implantada, y no había garantías de conservación a largo plazo.

Por lo tanto, no llegó a desarrollarse dadas las carencias y las necesidades técnicas y económicas que iba a suponer mantener de manera individual dicho programa por parte del ayuntamiento de Monforte del Cid, partiendo de la coyuntura económica de aquel entonces (año 2013).

Por otro lado, la Diputación Provincial de Alicante comenzó a desarrollar el Plan de Modernización de los ayuntamientos de la provincia de Alicante, Moderniza 6.0 (en la actualidad Moderniza 7.0), dirigido en primer lugar a municipios de menos de 20.000 habitantes, plan al que se adhirió el ayuntamiento de Monforte del Cid en el año 2013. Dicho plan consta de seis acciones (hoy en día cuenta con siete), siendo la Acción $4^{\mathrm{a}}$ la correspondiente a la Administración Electrónica.

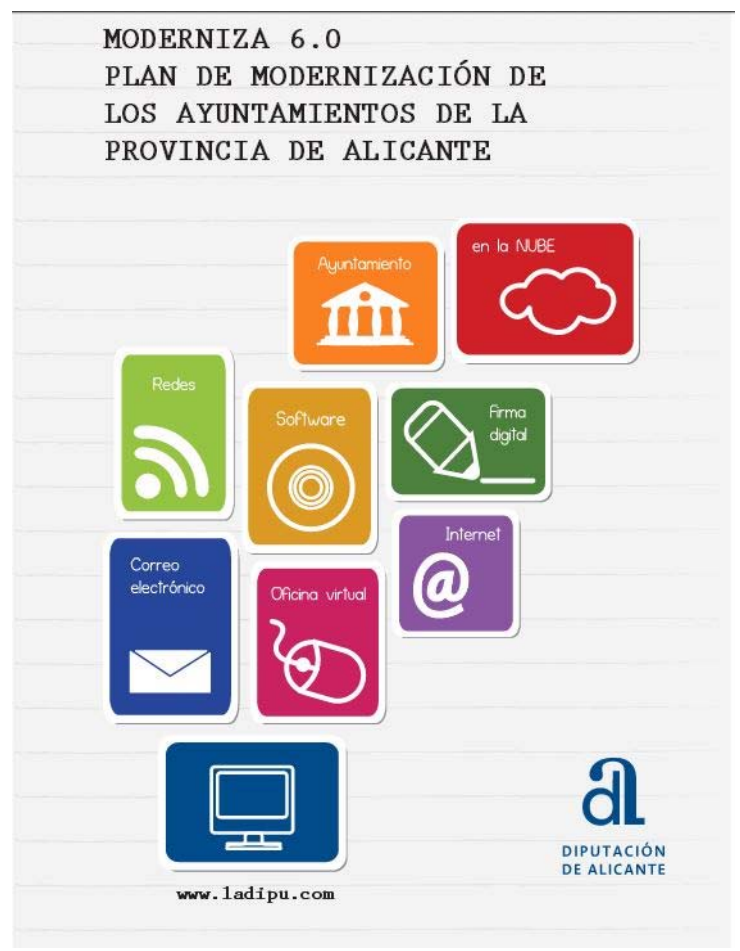

Figura 1. Moderniza 6.0 


\subsection{Plan de Modernización para ayuntamientos de la provincia de} Alicante: Acción $4^{a}$ Administración Electrónica: Gestiona

No vamos a entrar a detallar el funcionamiento de la plataforma Gestiona pero sí la experiencia en relación a su puesta en marcha y su funcionalidad, sobre todo en relación al archivo, partiendo de que éste ha sido el servicio responsable de su gestión e intermediario con los responsables de la plataforma que ya usan buena parte de los municipios de la provincia de Alicante.

En el año 2013, fecha de partida del Plan Moderniza, la empresa gestora hizo una consultoría en el ayuntamiento de Monforte del Cid de cara a identificar los distintos departamentos, el personal adscrito a cada uno de ellos y la presencia de unos requisitos técnicos mínimos para la implantación y desarrollo del proyecto. Estas especificaciones técnicas eran totalmente asumibles, por lo que con una serie de actualizaciones los terminales podían estar listos para la puesta en marcha de la plataforma de gestión de expedientes Gestiona.

El responsable, como ya hemos comentado, de la implantación en nuestro municipio ha sido la técnico de archivo. Desde luego no es habitual, y así nos lo hicieron ver los consultores, incluso comentaron que en la mayoría de los municipios donde habían implantado ya el programa no se habían encontrado con un servicio de archivo, o directamente lo habían dejado fuera (estamos hablando de ayuntamientos de menos de 20.000 habitantes). Pero los responsables del archivo de Monforte del Cid, no solo hemos estado vinculados al archivo histórico, sino que la tarea principal y más visible ha sido siempre el archivo administrativo, así como labores de gestión documental, tales como el diseño de bases de datos o pequeños SGD para los distintos departamentos.

Partiendo de aquí, el archivo ha sido en todo momento el servicio encargado del proyecto, desde el trámite para su adhesión al plan auspiciado por la Diputación de Alicante, como el intermediario con los técnicos de la empresa encargada de la implantación del programa. Dada esta responsabilidad también quedará la técnico del archivo como la responsable de configuración de la plataforma.

Con respecto al propio sistema, en relación al trámite del expediente electrónico y la asignación de firmas digitales, su manejo es muy sencillo. La interfaz no es complicada y es asequible para todo el mundo. Las ventajas son obvias: 
-Rapidez en la gestión del expediente

-Plataforma en la nube cuyo único requisito es un ordenador con conexión a INTERNET

-Auditoría permanente que permite localizar posibles "puntos negros" en la celeridad de la gestión de un expediente

-El contacto directo entre distintos departamentos en tiempo real

-Plataforma intuitiva y fácil de manejar

-Espublico es la empresa responsable de la plataforma, un ente de reconocido prestigio en relación a la provisión de modelos de procedimientos administrativos de la administración local, lo que en relación a la plataforma permite que el catálogo de procedimientos con el que cuenta sea muy completo y esté garantizada su actualización jurídica permanente

-Espacio de almacenamiento ilimitado y con plenas garantías de conservación, con certificado de conformidad con el Esquema Nacional de Seguridad (ENS)

Pero también son identificables los inconvenientes y muchas las mejoras a realizar:

-Mejorar el esquema de metadatos

-La organización del trabajo con expedientes abiertos, ya que es totalmente lineal

-Identificación de los expedientes que evite duplicidades

-Mayor normalización en los procesos

En relación al servicio de archivo como uno de los departamentos que usan la plataforma (ya no como responsable de la implantación del proyecto), por un lado tenemos la gestión de trámites en relación al archivo, como la consulta de documentos por parte de investigadores y ciudadanos en general, y por otro lado, como el servicio encargado de archivar los expedientes. El archivado, al ser un municipio pequeño es asumible por el personal del archivo (ya que no es algo habitual en esta fase del expediente), momento en que el programa nos permite incluir el código del cuadro de clasificación y la signatura. También podemos modificar los datos del procedimiento de cara a conseguir una mayor normalización en los puntos de acceso, pero de aquí parten las carencias: 
-No es posible asignar materias; las proporciona el programa si se escoge un procedimiento del catálogo que adjunta. En cualquier caso, es un listado de materias muy limitado:

-El cuadro con el que trabaja solo llega a nivel de sección, no al de serie, por lo que el archivero lo codificará manualmente

-No se plantean calendarios de conservación preceptivos en relación a aquellas series documentales ya valoradas y que incluyan el correspondiente dictamen vinculante emitido por la Junta calificadora de Documentos administrativos (JCDA)

-No permite la filtración ordenada de los expedientes archivados según criterios (cronológico, alfabético, numérico, etc.)

-El cuadro de búsqueda tiene muchas carencias, partiendo sobre todo que no es posible buscar documentos (solo expedientes y registros de entrada y salida, así como los documentos de los libros oficiales) ni existe un apartado que nos permita una búsqueda general

-La conservación de los documentos electrónicos, dado que éstos dependen de un entorno digital para ser legibles, no son independientes por sí mismos como los documentos en papel

Dado que el archivo asume el protagonismo en el mantenimiento y gestión del sistema, hemos establecido procedimientos paralelos para la gestión administrativa y cierre de los expedientes. Pero de cara a la conservación y correcta identificación de los mismos en municipios cuyos responsables no tengan esa visión archivística, porque simplemente el archivo haya quedado fuera del sistema o no exista el servicio, realmente puede llevar, por un lado, al ruido documental, y por otro, a poner en riesgo la preservación digital.

Recapitulando, el problema principal es la ausencia de la gestión de un archivo electrónico, ya que más allá del cierre del expediente y las garantías de conservación, como ya se ha indicado, no han planteado ni calendarios de conservación, ni plazos para transferencias. Con la integración de la gestión electrónica local en los servicios y aplicaciones de la Administración General del Estado, debemos presuponer que esto cambiará, ya que una de esas aplicaciones es precisamente ARCHIVE, herramienta del Estado para el archivo definitivo de expedientes y documentos electrónicos. 
En cualquier caso la plataforma está sujeta a continuos cambios y actualizaciones, y abierta a las sugerencias que se vayan planteando desde los distintos municipios adheridos al Plan de Modernización. Dentro de los cambios introducidos recientemente cabe destacar los siguientes:

-El portal de Transparencia, accesible a través de la Sede electrónica y configurado desde Gestiona al amparo de la Ley 19/2013, de 9 de diciembre, de Transparencia, Acceso a la información Pública y Buen Gobierno y la Ley 2/2015, de 2 de abril, de Transparencia, Buen Gobierno y Participación Ciudadana de la Comunitat Valenciana

-La última versión de la plataforma, Gestiona ENI, que incluye el certificado de conformidad con el Esquema Nacional de Seguridad y la adaptación al Esquema Nacional de Interoperabilidad. En relación al archivo, cabe resaltar la adaptación del nuevo catálogo de procedimientos a la Ley 39/2015, de 1 de octubre, del Procedimiento Administrativo Comín de las Administraciones Públicas y su disposición en Gestiona conforme a lo que sería un cuadro de clasificación archivístico; de hecho, el código de clasificación aparece ya reflejado y consignado desde el inicio del expediente. Por último, destacar también las facilidades que proporciona la nueva versión al estar preparada para la integración con las aplicaciones de la Administración General del Estado, como ya hemos comentado, y la normalización de los terceros.

\section{Conclusión}

Es indudable que en el entorno de la e-Administración y por tanto de los archivos electrónicos, se hace necesario más que nunca el trabajo multidisciplinar. La conservación de los archivos, sean digitales o en papel, sigue perteneciendo a los archiveros, pero debemos partir también de la propia génesis del documento, que ha variado por completo, y por lo tanto requiere por parte del archivo un reciclaje de cara a la comprensión de esa génesis, pues no olvidemos que no solo somos conservadores, sino también gestores.

Por lo tanto, el archivo debe seguir asumiendo su papel pero también reinventarse, y más que nunca estar presente en prácticamente todo el ciclo vital del documento. Por otro lado, es obvia la colaboración con otros profesionales como los informáticos, sin los cuales no sería posible la implantación de un sistema de gestión de documentos y la propia creación del archivo digital, ya que su desarrollo físico y lógico es acometida por ellos, siendo el archivero el 
responsable de marcar las pautas de descripción, la preservación y conservación del documento. Por último, también es necesaria la figura legal o en el caso de la administración local, la plena implicación de la Secretaría y/o técnicos de administración general. La autentificación y gestión de los documentos electrónicos necesitan de una supervisión y adaptación a todo un ámbito legislativo relativamente reciente en el terreno de la Administración Electrónica, siendo necesario contar con la supervisión de un responsable que asegure su aplicación.

Por último, y en relación a municipio de menos de 20.000 habitantes, la implantación de la e-Administración liderada por instituciones públicas como es el caso de la Diputación de Alicante, ahorra costes, garantiza la conservación de la documentación digital y normaliza procesos de gestión, salvaguardando las particularidades de cada municipio. En este sentido es donde debe incidir el servicio de archivo de la institución y hacerse más que nunca visible, ya que de nuestro trabajo en el presente dependerá que la entidad tenga un archivo histórico digital en el futuro.

\section{Bibliografía}

ALAMILLO DOMINGO, I., 2016. La regulación de la identificación, la firma y sello electrónicos en la Ley 39/2015. Consultor de los ayuntamientos y de los juzgados: Revista técnica especializada en administración local y justicia municipal, 24, 2766-2777. ISSN 0210-2161

CAMPOS ACUÑA, M. C. coord., 2016. El Nuevo Procedmiento local tras la Ley 39/2015. Madrid: Wolters Kluwer. ISBN 978-84-7052-713-5

DIPUTACIÓN PROVINCIAL DE ALICANTE, [sin fecha]. Memoria Plan Moderniza 7.0 : Plan Moderniza [en línea]. [Consulta: 13 diciembre 2017]. Disponible en: http://planmoderniza.diputacionalicante.es/memoria-plan-moderniza-7-0/

MÉNDEZ RODRÍGUEZ, E. M., 2003. La descripción de documentos electrónicos a través de metadatos: una visión para la archivística desde la nueva eAdministración. Revista d'Arxius, 2, 47-82. ISSN 1696-1552

MINISTERIO DE HACIENDA Y FUNCIÓN PÚBLICA, [sin fecha]. PAe Normas Técnicas [en línea]. [Consulta: 13 diciembre 2017]. Disponible en: https://administracionelectronica.gob.es/pae Home/pae Estrategias/pae In teroperabilidad Inicio/pae Normas tecnicas de interoperabilidad.html\#DO CUMENTOELECTRONICO 
MINISTERIO DE HACIENDA Y FUNCIÓN PÚBLICA, [sin fecha]. PAe Normas Técnicas [en línea]. [Consulta: 13 diciembre 2017]. Disponible en: https://administracionelectronica.gob.es/pae Home/pae Estrategias/pae In teroperabilidad Inicio/pae Normas tecnicas de interoperabilidad.html\#PO LITICAGESTION

MINISTERIO DE HACIENDA Y FUNCIÓN PÚBLICA, 2015. PAe - CTT General - ARCHIVE [en línea]. [Consulta: 13 diciembre 2017]. Disponible en: https://administracionelectronica.gob.es/ctt/archive\#.WjEZc6KYKeA

SERRA SERRA, J., 2005. Valoración y selección de documentos electrónicos: principios y aplicaciones. Revista TRLA, 12, 119-155. ISSN 1134-1602

SERRA SERRA, J., 2016. El archivo electrónico único en la Ley 39/2015: ¿Una oportunidad perdida? Consultor de los ayuntamientos y de los juzgados: Revista técnica especializada en administración local y justicia municipal, 24, 2788-2791. ISSN 02102161

VALMONACID LAMELAS, V. 2016. Ley de procedimiento ¿qué entra en vigor en 2018? Nosoloaytos [en línea]. [Consulta: 13 diciembre 2017]. Disponible en: https:// nosoloaytos.wordpress.com/2016/09/25/ley-de-procedimiento-queentra-en-vigor-en-2018/ 